\title{
An Estimation Method for Aircraft Longitudinal Static Stability Prior to Flight Test
}

\author{
Liu Chaoqiang \\ shanghai aircraft design \& research institute
}

\begin{abstract}
A quick method for estimating aircraft longitudinal static stability is developed based on the aircraft dimensions. Stick fixed and stick free stability estimation equations are reduced respectively, and the equations of stick deflection and stick force for flight tests are obtained. An application software was designed to make this estimation become practical. Three sorties of longitudinal static stability flight tests were planned and conducted to verify the software functions. The estimation results generally conformed to the flight test results.
\end{abstract}

\section{Keywords-Stick Fixed, Stick Free, Neutral Point}

\section{INTRODUCTION}

It's not uncommon that before flight tests of a new aircraft, the test pilot and flight test engineers (FTE) have no idea how much longitudinal stable the aircraft will be. The aircraft longitudinal static stability is not always available, or inaccurate, especially at marginal test conditions. Furthermore, the flight test team has to start the stability tests from normal condition and extend the envelope very slowly, which will increase the cost dramatically. Thereby, it is necessary to develop an method for estimating aircraft longitudinal static stability and the stability test results prior to flight test.

\section{ESTIMATION THEORY}

There are two ways that the static longitudinal stability of the aircraft is felt by the pilot. One way is through the variation required of the elevator angle and thereby the control position with change in airspeed. This variation is a function of the stability criterion $d C_{m} / d C_{L}$ with control locked and is usually referred to as the stick fixed longitudinal static stability. The other way is through the stick force required to change the speed of the aircraft from the trim point. For the aircraft with a reversible flight control system, that stability relates to control left to float free with the wind and is commonly referred to as stick free longitudinal static stability.

\section{A. Stick Fixed Estimation}

For most symmetric airplanes, the longitudinal equilibrium equations or equations of motion can be considered independent of lateral and directional equations. The main pitch moments on the aircraft are presented in Figure 1. The non-dimensional coefficient summation of all contribution parts about the airplane's c.g. is obtained as shown in equation (2-1).

$$
\mathrm{C}_{m}=C_{N} \frac{x_{a}}{c}+C_{c} \frac{z_{a}}{c}+C_{\text {mac }}+C_{m f u s}+C_{\text {mact }} \frac{S_{t} c_{t}}{S_{w} c} \eta_{t}+C_{c t} \frac{S_{t} h_{t}}{S_{w} c} \eta_{t}-C_{N t} \frac{S_{t} l_{t}}{S_{w} c} \eta_{t}
$$

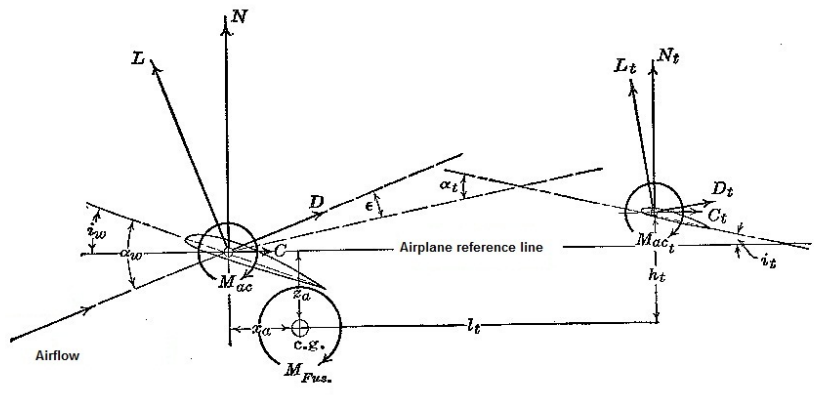

Figure 1. Forces and Moments of the Airplane

The fifth and sixth terms of equation (1) are relative small and negligible, and therefore be eliminated from the further estimation. The angle of attack is the major importance for light aircraft longitudinal static stability with low cruise Mach number, which is proportional to the lift coefficient. The slope of pitching moment coefficient against lift coefficient $d C_{m} / d C_{L}$ is used for evaluating the aircraft longitudinal static stability, as shown in equation (2).

$$
\frac{\mathrm{dC}_{m}}{\mathrm{dC}_{\mathrm{L}}}=\frac{d C_{N}}{d C_{L}} \frac{x_{a}}{c}+\frac{d C_{c}}{d C_{L}} \frac{z_{a}}{c}+\frac{d C_{m f u s}}{d C_{L}}-\frac{d C_{N t}}{d C_{L}} \frac{S_{t} l_{t}}{S_{w} c} \eta_{t}
$$

A negative sign of this derivative is required for static stability. The more negative the more stable. Positive sign indicates instability of the aircraft. At neutral stability, the derivative is zero.

There are four major parts of contribution to the derivative as can be seen from equation (2). Their estimation theories are discussed separately.

a) Wing contribution. The first two terms of equation (2) come from wing contribution. For quick estimation prior to flight test, the drag contribution is eliminated and the total wing contribution becomes a very simple equation:

$$
\left(\frac{\mathrm{dC}_{m}}{\mathrm{dC}_{\mathrm{L}}}\right)_{\text {wing }}=\frac{x_{a}}{c}=x_{c g}-x_{a c}
$$

Wing aerodynamic center usually locates on the quarter chord position. So the wing contribution mainly depends on the C.G. position.

b) Tail contribution. The tail contribution to the stability reduced is:

$$
\left(\frac{d C_{m}}{d C_{L}}\right)_{t}=-\frac{a_{t}}{a}\left(1-\frac{d \varepsilon}{d \alpha}\right) \bar{V}
$$


The rate of change of downwash $d \varepsilon / d \alpha$, airfoil lift coefficient slope $a_{0}$, wing and tail lift coefficient slopes $a$ and $a_{t}$ can be estimated using experimental estimation equations, with aircraft dimensions. As can be seen from equation (4), the tail stability contribution is usually negative, which is stabilizing.

c) Fuselage contribution. The fuselage contribution to the longitudinal static stability of the aircraft is always destabilizing. The theory accounting for the effects of the fuselage on the stability is rather complex as the interference effects of the wing and its flow pattern are very hard to predict. A simple estimation method is to use:

$$
\left(\frac{d C_{m}}{d C_{L}}\right)_{\text {Fus }}=\frac{K_{f} w_{f}^{2} L_{f}}{S_{w} c a_{w}}
$$

The fuselage stability coefficient ${ }^{K_{f}}$ is an empirical factor developed from experimental evidence. It is reliable for estimating normal light aircraft.

d) Power Effects. The stability equation (2) does not consider the contribution of power effects. But if there is a distance between c.g. and thrust line, the thrust change will produce a moment about the pitching axis and affect the longitudinal static stability.

The power output of a piston or turbo-prop engine $P$ can be considered constant with speed $v$, i.e. $P=T \cdot v$. The propeller power contribution is:

$$
\frac{\mathrm{dC}_{m T}}{\mathrm{dC}_{\mathrm{L}}}=\frac{3 \mathrm{P} \rho^{\frac{1}{2}} \mathrm{~S}^{\frac{1}{2}}}{2^{\frac{3}{2}} \mathrm{~W}^{\frac{3}{2}} \mathrm{z}} \mathrm{zC}_{\mathrm{L}}^{\frac{1}{2}}
$$

The thrust of a jet engine can be considered constant with speed. Its contribution to static longitudinal stability is:

$$
\frac{\mathrm{dC}_{m T}}{\mathrm{dC}_{\mathrm{L}}}=\frac{T z}{\mathrm{~W} c}
$$

The equation (6) and (7) indicate that the thrust and weight will affect the power contribution to static longitudinal stability. If the aircraft c.g. is below the thrust line, or $Z$ is negative, the power is stabilizing.

Combine all the contributions discussed above, the stick fixed longitudinal static stability estimating equation becomes:

$\frac{d C_{m}}{d C_{L}}=\left(x_{c g}-x_{a c}\right)+\left(\frac{d C_{m}}{d C_{L}}\right)_{F u s}+\frac{d C_{m T}}{d C_{L}}-\frac{\bar{V} a_{t}}{a}\left(1-\frac{d \varepsilon}{d \alpha}\right)$

At the neutral point, the aircraft is neutrally stable and the derivative $d C_{m} / d C_{L}$ equals zero. From equation (8), by reducing the derivative to zero, the neutral point $N_{0}$ can be determined:

$$
N_{0}=x_{c g}=x_{a c}-\left(\frac{d C_{m}}{d C_{L}}\right)_{F u s}-\frac{d C_{m T}}{d C_{L}}+\frac{\bar{V} a_{t}}{a}\left(1-\frac{d \varepsilon}{d \alpha}\right)
$$

The stability at any other c.g. position might be determined by subtracting the neutral point $N_{0}$ from the c.g. location ${ }^{x_{c g}}$, although the tail contribution may reduce the accuracy.

At the trim condition, the pitch moment is zero. Apply elevator deflection $d \delta_{e}$, the pitch moment becomes:

$$
C_{m}=\left(x_{c g}-x_{a c}\right) C_{L}+\left(C_{m}\right)_{F u s}+\left(C_{m T}\right)-\bar{V} a_{t}\left(\alpha_{t}+\tau d \delta_{e}\right)
$$

Then the elevator position at any airspeed point can be estimated:

$$
\delta_{e}=\delta_{e-t r i m}-\frac{d C_{m}}{d C_{L}} \cdot \frac{2 W}{\rho S \bar{V} \tau a_{t}} \cdot\left(\frac{1}{v^{2}}-\frac{1}{v_{\text {trim }}^{2}}\right)
$$

With the gearing $m$ of the stick deflection to elevator angle deflection, the stick position can be determined according to:

$$
\delta_{s}=\delta_{s-t r i m}-m \cdot \frac{d C_{m}}{d C_{L}} \cdot \frac{2 W}{\rho S \bar{V} \tau a_{t}} \cdot\left(\frac{1}{v^{2}}-\frac{1}{v_{\text {trim }}{ }^{2}}\right)
$$

With stability estimated and test condition parameters, the stick position of the tests can be plotted against equivalent airspeed according to the (12).

\section{B. Stick Free Estimation}

For the aircraft with reversible elevator control system, the elevator will float with or against the wind, depending on the pressure distribution over the elevator. The change in stability on freeing the elevator is all contained in the tail contribution and is a function of the floating characteristics of the elevator and therefore of the elevator hinge moment. Under the assumption that the variation of the hinge moment with angle of attack and deflection is linear, the elevator hinge moment coefficient can be expressed in terms of partial derivatives as shown in (13).

$$
C_{H}=C_{H \alpha} \alpha_{\mathrm{T}}+C_{H \delta} \delta_{e}
$$

The stick free longitudinal static stability estimation equation is reduced.

$$
\frac{d C_{m}}{d C_{L}}=\left(x_{c g}-x_{a c}\right)+\left(\frac{d C_{m}}{d C_{L}}\right)_{F u s}+\frac{d C_{m T}}{d C_{L}}-\bar{V} \frac{a_{t}}{a}\left(1-\frac{d \varepsilon}{d \alpha}\right)\left(1-\tau \frac{C_{H \alpha}}{C_{H \delta}}\right)
$$

The hinge moment coefficient with angle of attack and elevator deflection $C_{H \alpha}$ and $C_{H \delta}$ is normally negative. The estimating method of $C_{H \alpha}$ and $C_{H \delta}$ in the software uses the empirical results.

If the aircraft's flight control system is irreversible, the elevator hinge moment coefficient does not change with angle of attack and elevator deflection, therefore $C_{H \alpha}$ and $C_{H \delta}$ are both zero. Both the stick deflection and stick force are then proportional to the elevator angle deflection. Then, the stick free static stability equation (14) is no applicable.

By reducing the derivative $d C_{m} / d C_{L}$ to zero according to equation (14), the stick free neutral point $N_{0}{ }^{\prime}$ is obtained.

$$
N_{0}{ }^{\prime}=x_{a c}-\left(\frac{d C_{m}}{d C_{L}}\right)_{F u s}-\frac{d C_{m T}}{d C_{L}}+\bar{V} \frac{a_{t}}{a}\left(1-\frac{d \varepsilon}{d \alpha}\right)\left(1-\tau \frac{C_{H \alpha}}{C_{H \delta}}\right)
$$

During the stick free stability flight test, the stick forces required to change the airspeed of the aircraft from the trim point should be tested. The stick free stability criterion can be obtained from the gradient of stick force versus speed curve through trim. The stick force required by the pilot can be written as follows.

$$
F_{S}=1 / 2 m C_{H} \rho v^{2} S_{e}
$$

The stick force at any airspeed can be determined by equation (17) 


$$
F_{S}=\frac{m W S_{e} C_{H \delta}}{\overline{V \tau} a_{t} S_{w}}\left(\frac{d C_{m}}{d C_{L}}\right)_{\text {free }}\left[1-\left(\frac{v}{v_{\text {trim }}}\right)^{2}\right]
$$

The stick force at a given test airspeed depends on the stick free stability, which is obtained from equation (14), as well as the test condition, elevator moment coefficient, gearing from elevator to stick force, etc.

For aircraft with irreversible flight control system, equation (17) is not applicable to stick force estimation. However, equation (12) could be used instead by changing the gearing of stick force to elevator deflection ( $\mathrm{Kg} /$ degree).

\section{SOFTWARE STRUCTURE}

Based on the estimation method reduced above, a software was designed to estimate the aircraft longitudinal static stability and its flight test. The functional flow chart is as shown in Figure 2. The software consists of six major forms: Main form, Input form, Stick Fixed Estimation form, Stick Free Estimation form, Stick Deflection form and Stick Force form. A series of accessary tools are designed for increasing the practicability of estimating prior to fligh test.

When the aircraft dimensions (twenty parameters) are added to the input form, the stick fixed and stick free longitudinal statc stability estimation results will display, and the stick position and stick force estimation results will be computed when the test conditions are added. The stick fixed stability estimation results include dozens of aerodynamic parameters, longitudinal stability contributions, neutal point, etc.

\section{Flight TEST}

The test objective was to verify the software function and determine the aircraft longitudinal static stability. The test aircraft included Cessna172 (ZS-MWL) and JABIRU (ZU-DAX). They had reversible elevator control systems. The tests were conducted from 5th Nov, 2010 to 12 Jan 2011 at Oudtshoorn airfield in South Africa. The test matrix is listed in Table 1.

The test results are compared with the estimation results. The flight test results of these test points and the estimation results were very close. One example of stick position test of Cessna-172 at forward CG is presented in Figure 3. Observation of Figure 3 shows that, the estimated stick position with equivalent airspeed curve had the same decreasing trend as flight test result. The break out frictions caused a little drift of test point from the trim position, which did not affect the stability test results. The estimating slope was slightly flatter than the test results. However, the slope difference was correspondingly small, with no more than 5 percent stick position errors as can be seen from the Figure 3. The estimation results of other test conditions also had small errors from fligh test results, with less than 10 percent. The estimation results were close to the flight test results with insignificant errors, thereby the estimation results of the aircrafts' longitudinal static stability and its tests were satisfactory.

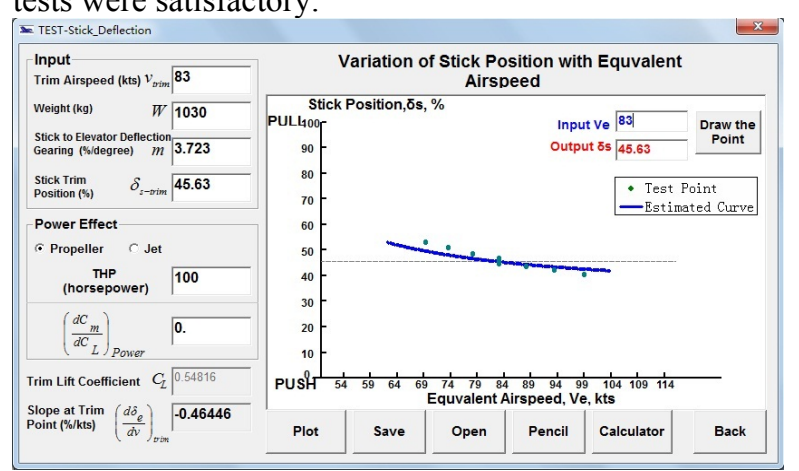

Figure 3. Stick Deflection Estimation of Cessna172 at Forward C.G

\section{SUMMARIES}

An estimation method of aircraft longitudinal static stability has been developped. The estimation equations of stick fixed and stick free longitudinal static stability have been reduced, and the equations of stick position and stick force for the stability flight test have been obtained. A software has been designed based on these estimation equations for estimating prior to flight test, with twenty dimensional parameters input. The major aerodynamic parameters related to every longitudinal static stability contributions can be obtained and used to analyse their effects to the stability contributions. The accessary tools can increase the practicability of estimation prior to fligh test. Three sorties have been condcuted on the light aircraft Cessna-172 (ZS-MWL) and JABIRU (ZU-DAX) to verify the estimation functions. The flight test results indicated that the estimation results had insignificant difference from the test results.

\section{REFERENCES}

[1] Contrland D. Perkins and Robert E.Haye. Airplane Performance Stability and Control. Published by John Wiley and Sons

[2] Barnes W. McCormick. Aerodynamic, Aeronautics and Flight Mechanics. Published by John Wiley and Sons

[3] U.S. NAVAL TEST PILOT SCHOOL FLIGHT TEST MANUAL FIXED WING STABILITY AND CONTROL. Commanding Officer. Patuxent River. 


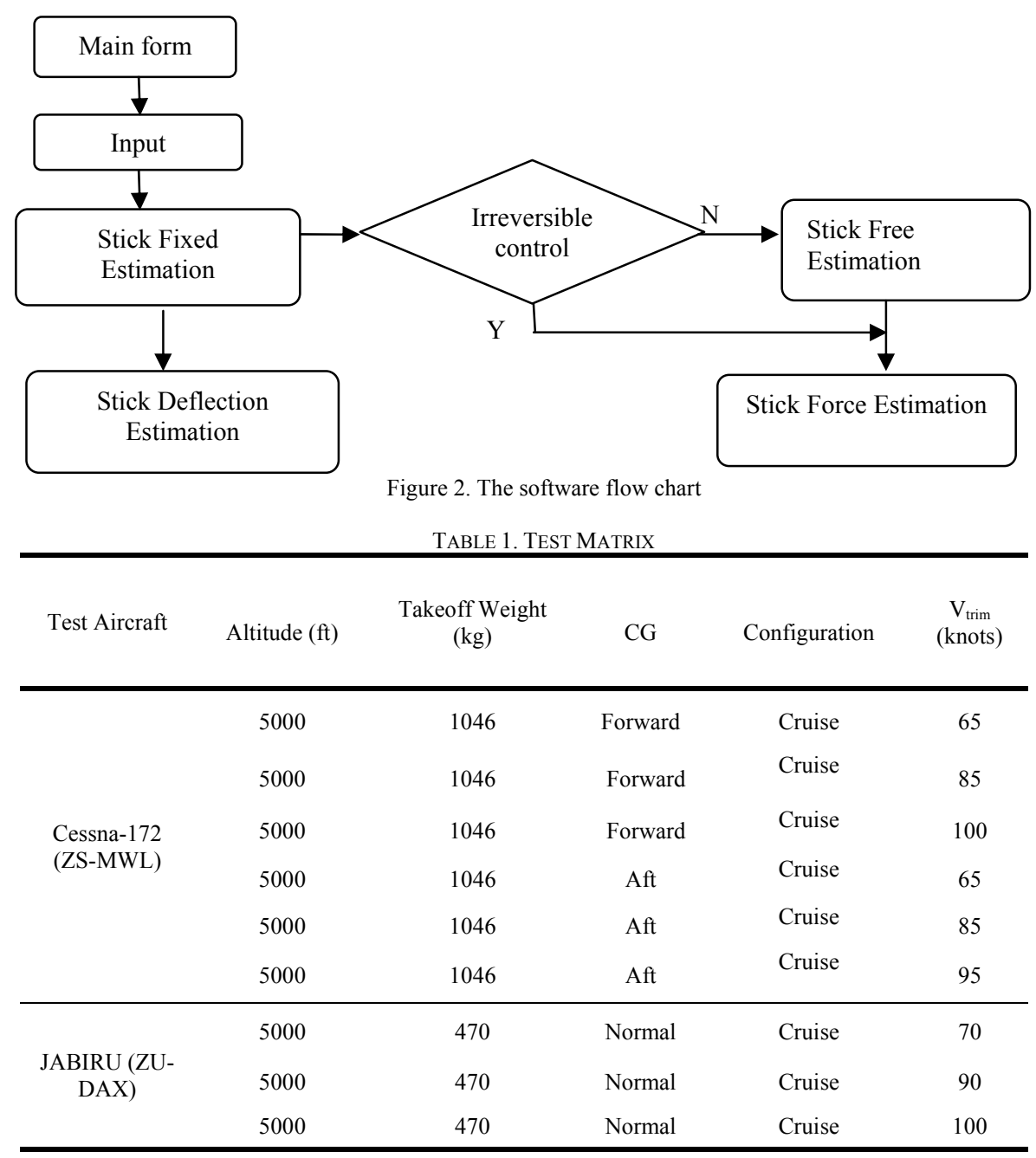

